\title{
Efficacy of acupuncture for sciatica: study protocol for a randomized controlled pilot trial
}

\author{
Fang-Ting Y ${ }^{1,2}$, Guang-Xia Ni ${ }^{3}$, Guo-Wei Cai ${ }^{4}$, Wen-Jun Wan ${ }^{5}$, Xiao-Qing Zhou ${ }^{6}$, Xiu-Li Meng ${ }^{7}$, Jin-Ling Li ${ }^{1,2}$, \\ Jian-Feng Tu ${ }^{1,2}$, Li-Qiong Wang ${ }^{1,2}$, Jing-Wen Yang ${ }^{1,2}$, Hai-Yang Fu ${ }^{8}$, Xin-Chang Zhang ${ }^{3}$, Jing Li ${ }^{4}$, Yan-Fu Wang ${ }^{5}$, \\ Beng Zhang ${ }^{6}$, Xiao-Hui Zhang ${ }^{9}$, Hao-Lin Zhang ${ }^{9}$, Guang-Xia Shi ${ }^{1,2^{*}}$ and Cun-Zhi Liu ${ }^{1,2,10}$
}

\begin{abstract}
Background: Acupuncture is widely used for pain diseases while evidence of its efficacy for sciatica is insufficient. We aim to explore the feasibility and efficacy of acupuncture with different acupoint selecting strategies for sciatica induced by lumbar disc herniation.

Methods: This is a multicenter, three-arm, patient-assessor-blinded randomized controlled pilot trial. Ninety patients will be assigned randomly into 3 groups including disease-affected meridians (DAM) group, non-affected meridians (NAM) group, and sham acupuncture (SA) group in a 1:1:1 ratio. The trial involves a 4-week treatment along with follow-up for 22 weeks. The primary outcome is the change of leg pain intensity measured by the visual analogue scale (VAS) from baseline to week 4 after randomization. Secondary outcomes include functional status, back pain intensity, and quality of life. Adverse events will also be recorded.
\end{abstract}

Discussion: The results will inspire the optimal acupuncture strategy for sciatica and help establish a better design as well as power calculation for a full-scale study.

Trial registration: ChiCTR2000030680 (Chinese Clinical Trial Registry, http://www.chictr.org.cn, registered on 9 March 2020).

\section{Background}

Sciatica is characterized by radiating leg pain along the course of the sciatic nerve sometimes accompanied by back pain and neurological deficits [1]. The prevalence ranges from 1.2 to $43 \%$ globally based on controversial definitions [2]. Lumbar disc herniation is the leading cause of $85 \%$ of patients with sciatica [3]. Sciatica affects daily life and productivity and consumes more health resources when compared to low back pain [4].

\footnotetext{
* Correspondence: shiguangxia2008@126.com

'International Acupuncture and Moxibustion Innovation Institute, Beijing University of Chinese Medicine, Beijing 100029, China

${ }^{2}$ Acupuncture Research Center, School of Acupuncture-Moxibustion and Tuina, Beijing University of Chinese Medicine, Beijing 100029, China Full list of author information is available at the end of the article
}

Conservative treatments are the first-line options for sciatica [5, 6]. Medicine and epidural steroid injection are commonly used although long-term benefits are uncertain and side effects (e.g., headache and dizziness) or complications (e.g., epidural hematoma) occur sometimes [7-9]. Most pain and related disabilities could resolve in weeks [5], but up to $30 \%$ of patients were reported with pain lasting for 1 year or longer [10]. Therefore, long-term effective and safe conservative treatments might be potential solutions.

Acupuncture has been widely used for pain diseases. Meta-analysis indicated that acupuncture had a persistent effect on 4 chronic pains which decreased only $15 \%$ after 1 year [11]. However, high-quality evidence remains

C C The Author(s). 2021 Open Access This article is licensed under a Creative Commons Attribution 4.0 International License, which permits use, sharing, adaptation, distribution and reproduction in any medium or format, as long as you give appropriate credit to the original author(s) and the source, provide a link to the Creative Commons licence, and indicate if changes were made. The images or other third party material in this article are included in the article's Creative Commons licence, unless indicated otherwise in a credit line to the material. If material is not included in the article's Creative Commons licence and your intended use is not permitted by statutory regulation or exceeds the permitted use, you will need to obtain permission directly from the copyright holder. To view a copy of this licence, visit http://creativecommons.org/licenses/by/4.0/ The Creative Commons Public Domain Dedication waiver (http://creativecommons.org/publicdomain/zero/1.0/) applies to the data made available in this article, unless otherwise stated in a credit line to the data. 
scarce for acupuncture treating sciatica [12, 13]. Acupoints play a key role in treatment that different acupoint selecting strategies could generate discrepant effects $[14,15]$. To optimize acupuncture strategy for sciatica, this pilot study will evaluate feasibility and efficacy of acupuncture with different acupoint selecting methods. The hypothesis is that acupuncture on diseaseaffected meridians might have better effects. Reliable data will be used to develop a better design including sample size calculation for a further randomized controlled trial (RCT).

\section{Methods/design}

\section{Study design}

The detailed study process is illustrated in Fig. 1. We design this multicenter, parallel-group, patient-assessor blinded RCT following the Consolidated Standards of Reporting Trials (CONSORT) and the Standards for Reporting Interventions in Clinical Trials of Acupuncture (STRICTA) guidelines $[16,17]$. The trial will be conducted in outpatient departments of 6 hospitals in 4 cities in China, including the Department of acupuncture, Union Hospital, Union Hospital, Tongji Medical College, Huazhong University of Science and Technology; the Department of acupuncture, Affiliated Hospital of Nanjing University of Chinese Medicine; the Department of Rehabilitation, the Central Hospital of Wuhan, Tongji Medical College, Huazhong University of Science

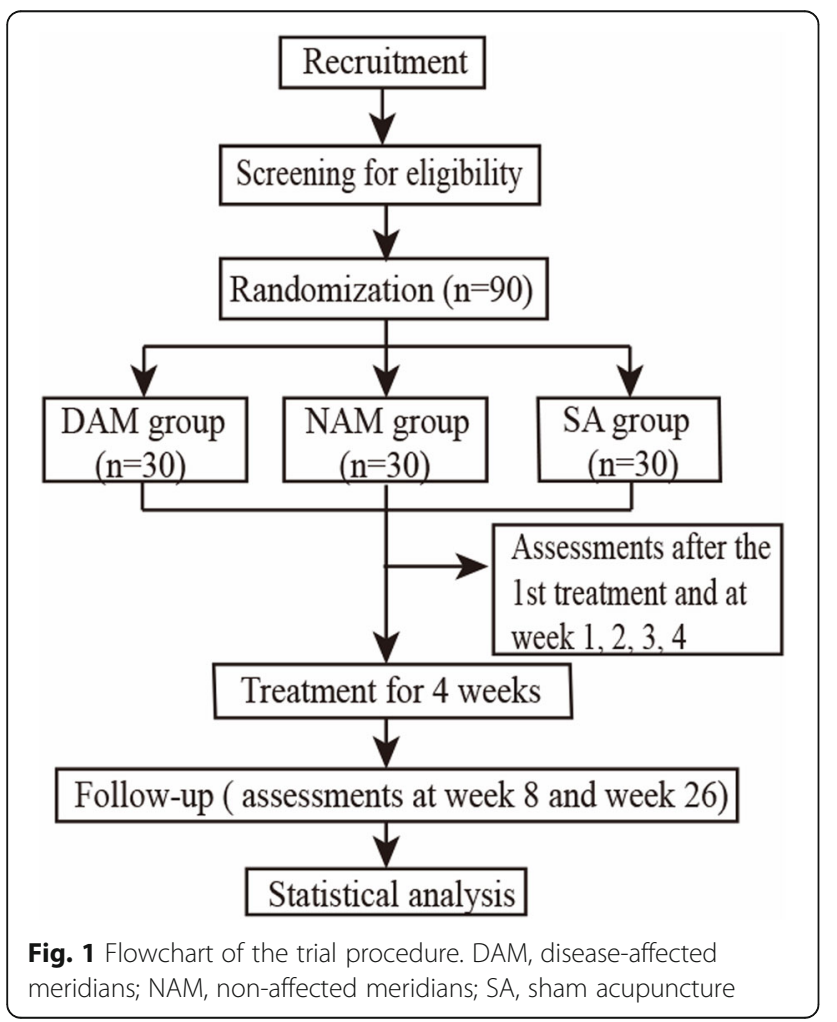

and Technology; the Department of acupuncture, Shenzhen Hospital, Beijing University of Chinese Medicine; the Department of pain medicine center and traditional Chinese medicine, Peking University Third Hospital; and the Department of acupuncture, Dongzhimen Hospital Affiliated to Beijing University of Chinese Medicine. The Research Ethics Committee of Beijing University of Chinese Medicine has approved the study protocol (version 2.0, 13 January 2020) with an approval number of 2020BZHYLL0105. It was registered at the Chinese Clinical Trial Registry (http://www.chictr.org.cn) on 9th March 2020 with the registration number of ChiCTR2000030680. The protocol is reported in accordance with the Standard Protocol Items (SPIRIT) (Additional file 1).

\section{Patients}

There will be 6 centers conducting this study to ensure recruitments where many patients visit doctors for sciatica every day. Eligible patients will be recruited from outpatient clinics through advertisements on posters, newspapers, and WeChat. To promote recruitment as well as compliance, all treatment during the study will be offered freely.

\section{Inclusion criteria}

The study will include both male and female patients (1) aged between 18 and 70 years; (2) having symptoms of sciatica for 1 month or longer from the onset, diagnosed as unilateral radiating leg pain below the knee with positive straight-leg raise test or at least one neurological deficit symptoms as paraesthesia, muscle weakness, or reflex abnormalities. The imaging evidence of lumbar disc herniation is requested on either magnetic resonance imaging (MRI) or computed tomography (CT); (3) leg pain intensity for $40 \mathrm{~mm}$ or more measured by the visual analogue scale (VAS) [18].

\section{Exclusion criteria}

Patients will be excluded if they meet any of the following criteria: (1) patients who have or are suspected to have severe spinal diseases (e.g., cauda equina syndrome) or progressive neurological symptoms (e.g., progressive muscle weakness); (2) patients with sciatica caused by other conditions than lumbar disc herniation; (3) patients who have undergone surgery for lumbar disc herniation within the past 6 months; (4) patients who plan to have spinal surgery or other interventional therapies during the first 4 weeks of the trial; (5) patients continually taking antiepileptic medication, antidepressant medication, opioids or corticosteroids; (6) patients who have cardiovascular, liver, kidney, or hematopoietic system diseases, mental health disorders, or other severe coexisting diseases (e.g., cancer); (7) patients who are 
pregnant, breastfeeding, or planning to conceive during the trial; (8) patients who received acupuncture therapy within the past 6 months.

\section{Dropout criteria}

Patients will be removed if they are unwilling to continue participation. The reasons for dropouts will be recorded.

\section{Randomization}

An independent statistician will generate the random sequence by the central block randomization method using IBM SPSS Statistics, version 21.0 (International Business Machines Corporation, China). Eligible patients will be assigned randomly into the disease-affected meridians (DAM) group, non-affected meridians (NAM) group, and sham acupuncture (SA) in a 1:1:1 ratio. An independent researcher in charge of informing assignment will implement the allocation schedule through a centralized telephone randomization procedure. To ensure the allocation concealment, acupuncturists will make a call to ask for the information of assignment before the first treatment. The independent researcher will send messages containing allocation details immediately. Before randomization, trial information will be provided to each eligible patient. Researchers (doctors) will get written informed consent from eligible patients who are willing to participate.

\section{Blinding}

Patients, outcome assessors, and statistician will be blinded to the assignment. Differences between groups present mainly at point selections and acupuncture performance. Blunt-tipped needles for blinding will be similar to conventional needles. Adhesive foam pads will be put on treating points to cover the difference in acupuncture performance (Fig. 2).

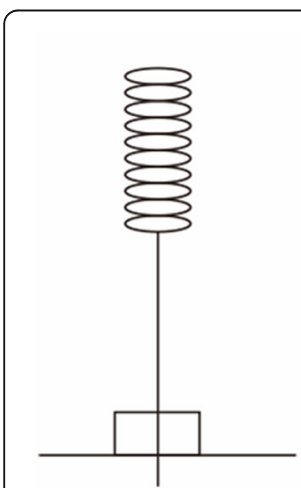

acupuncture

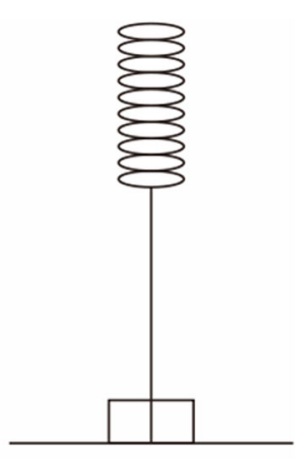

sham acupuncture
Fig. 2 Schematic diagram of acupuncture and sham acupuncture

\section{Interventions}

All patients will receive advice for managing sciatica in daily life such as remaining active, using a hard bed, or losing weight. Based on the assignment, they will receive 12 sessions of 30 -min treatments over 4 weeks (ideally 3 times a week with an interval of 1 or 2 days). Acupoints are predefined based on experienced acupuncturists' consensus and literature review [19]. Locations of acupoints are all according to the WHO Standard Acupuncture Locations shown in Tables 1 and 2 and Fig. 3 [20]. Patients could ask for Celebrex (Pfizer Pharmaceutical Co., Ltd) from researchers with guidance, and the use will be recorded in detail.

\section{DAM group}

Semi-standardized treatment will be provided with acupoints on affected meridians in this group. There will be four obligatory acupoints and five adjunct acupoints. Obligatory acupoints are bilateral Dachangshu (BL25) and Guanyuanshu (BL26). Depending on the distribution of the pain, sciatica localized at the side of the affected leg will be treated with adjunct acupoints on gallbladder meridian including Huangtiao (GB30), Fengshi (GB31), Xiyangguan (GB33), Yanglingquan (GB34), and Xuanzhong (GB39). Sciatica localized at the back of the affected leg will be treated with adjunct acupoints on bladder meridian including Zhibian (BL54), Chengfu (BL36), Weizhong (BL40), Chengshan (BL57), and Kunlun (BL60). For patients with pain in both side and back, adjunct acupoints will be selected from the ten adjunct acupoints above based on the personal experiences of acupuncturists.

After strict disinfection of the skin around acupoints and acupuncturists' hands using 75\% alcohol, adhesive foam pads (10-mm diameter and 5-mm height) will be placed on all acupoints with patients in a prone position. Disposable stainless steel acupuncture needles $(0.30 \times 75$ mm, Suzhou Huatuo Medical Instrument Co, Ltd) will be inserted in BL25 and BL26 on the affected side deeply to $40-70 \mathrm{~mm}$. After performing twirling, lifting, and thrusting manipulations for about $10 \mathrm{~s}$, patients are expected to have "de qi" sensation (a complex feeling including soreness, numbness, heaviness, distention and dull pain, etc.) radiating down to the affected leg. Other acupoints will be treated with needles $(0.30 \mathrm{~mm} \times 40 / 50 /$ $75 \mathrm{~mm}$ ) inserted to normal depths to reach "de qi" sensation locally. All needles will be retained for $30 \mathrm{~min}$.

\section{NAM group}

Nine acupoints on non-affected meridians will be used as follows: bilateral Yaoyan (EX-B7) and Pigen (EX-B4) and unilateral Yinbao (LR9), Ququan (LR8), Ligou (LR5), Fuliu (KI7), and Gongsun (SP4) on the affected leg. All acupoints will be treated by needles $(0.30 \times 40 /$ 
Table 1 Location of acupoints in DAM group

\begin{tabular}{|c|c|}
\hline Acupoints & Location \\
\hline Dachangshu (BL25) & $\begin{array}{l}\text { In the lumbar region, at the same level as the inferior border of the spinous process of the } \\
\text { fourth lumbar vertebra (L4), } 1.5 \text { cun lateral to the posterior median line. }\end{array}$ \\
\hline Guanyuanshu (BL26) & $\begin{array}{l}\text { In the lumbar region, at the same level as the inferior border of the spinous process of the } \\
\text { fifth lumbar vertebra (L5), } 1.5 \text { cun lateral to the posterior median line }\end{array}$ \\
\hline Huantiao (GB30) & $\begin{array}{l}\text { In the buttock region, at the junction of the lateral one third and medial two thirds of the } \\
\text { line connecting the prominence of the greater trochanter with the sacral hiatus. }\end{array}$ \\
\hline Fengshi (GB31) & $\begin{array}{l}\text { On the lateral aspect of the thigh, in the depression posterior to the iliotibial band where } \\
\text { the tip of the middle finger rests, when standing up with the arms hanging alongside the thigh. }\end{array}$ \\
\hline Xiyangguan (GB33) & $\begin{array}{l}\text { On the lateral aspect of the knee, in the depression between the biceps femoris tendon } \\
\text { and the iliotibial band, posterior and proximal to the lateral epicondyle of the femur. }\end{array}$ \\
\hline Yanglingquan (GB34) & On the fibular aspect of the leg, in the depression anterior and distal to the head of the fibula. \\
\hline Xuanzhong (GB39) & $\begin{array}{l}\text { On the fibular aspect of the leg, anterior to the fibula, } 3 \text { cun proximal to the prominence of } \\
\text { the lateral malleolus. }\end{array}$ \\
\hline Zhibian (BL54) & $\begin{array}{l}\text { In the buttock region, at the same level as the fourth posterior sacral foramen, } 3 \text { cun lateral } \\
\text { to the median sacral crest. }\end{array}$ \\
\hline Chengfu (BL36) & In the buttock region, at the midpoint of the gluteal fold. \\
\hline Weizhong (BL40) & On the posterior aspect of the knee, at the midpoint of the popliteal crease. \\
\hline Chengshan (BL57) & $\begin{array}{l}\text { On the posterior aspect of the leg, at the connecting point of the calcaneal tendon with } \\
\text { the two muscle bellies of the gastrocnemius muscle. }\end{array}$ \\
\hline Kunlun (BL60) & $\begin{array}{l}\text { On the posterolateral aspect of the ankle, in the depression between the prominence of } \\
\text { the lateral malleolus and the calcaneal tendon. }\end{array}$ \\
\hline
\end{tabular}

One "cun" is defined as the width of the interphalangeal joint of patient's thumb

$50 \mathrm{~mm}$ ) inserted into the skin with manipulations later to reach the "de qi" sensation. Other treatment settings are the same as those for the DAM group.

\section{SA group}

We select nine non-acupoints in this group with locations shown in Table 3. They are localized in the middle of the gallbladder meridian and bladder meridian as one selecting method for non-acupoints [21]. Blunt-tipped needles $(0.30 \times 25 \mathrm{~mm})$ will be inserted only into the pad with no penetration into the skin. No "de qi" sensation is required in this group and other settings will be the same.

\section{Outcomes}

The primary outcome is defined as the change of leg pain intensity on VAS from baseline to week 4 measuring pain over the prior $24 \mathrm{~h}$. The VAS presents as a 0 $100 \mathrm{~mm}$ ruler with 0 representing no pain and 100 representing unbearable pain. The specific score will be determined by the distance from 0 to the patient's mark.

Table 2 Location of acupoints in NAM group

\begin{tabular}{|c|c|}
\hline Acupoints & Location \\
\hline Yaoyan (EX-B7) & $\begin{array}{l}\text { In the lumbar region, at the same level as the inferior border of the spinous process of } \\
\text { the fourth lumbar vertebra }(L 4), 3.5 \text { cun lateral to the posterior median line. }\end{array}$ \\
\hline Pigen (EX-B4) & $\begin{array}{l}\text { In the lumbar region, at the same level as the inferior border of the spinous process of } \\
\text { the first lumbar vertebra }(L 1), 3.5 \text { cun lateral to the posterior median line. }\end{array}$ \\
\hline Yinbao (LR9) & $\begin{array}{l}\text { On the medial aspect of the thigh, between the gracilis and the sartorius muscles, } 4 \text { cun } \\
\text { proximal to the base of the patella. }\end{array}$ \\
\hline Ququan (LR8) & $\begin{array}{l}\text { On the medial aspect of the knee, in the depression medial to the tendons of the } \\
\text { semitendinosus and the semimembranosus muscles, at the medial end of the popliteal } \\
\text { crease. }\end{array}$ \\
\hline Ligou (LR5) & $\begin{array}{l}\text { On the anteromedial aspect of the leg, at the center of the medial border (surface) of } \\
\text { the tibia, } 5 \text { cun proximal to the prominence of the medial malleolus. }\end{array}$ \\
\hline Fuliu (KI7) & $\begin{array}{l}\text { On the posteromedial aspect of the leg, anterior to the calcaneal tendon, } 2 \text { cun superior } \\
\text { to the prominence of the medial malleolus. }\end{array}$ \\
\hline Gongsun (SP4) & $\begin{array}{l}\text { On the medial aspect of the foot, anteroinferior to the base of the first metatarsal bone, } \\
\text { at the border between the red and white flesh. }\end{array}$ \\
\hline
\end{tabular}



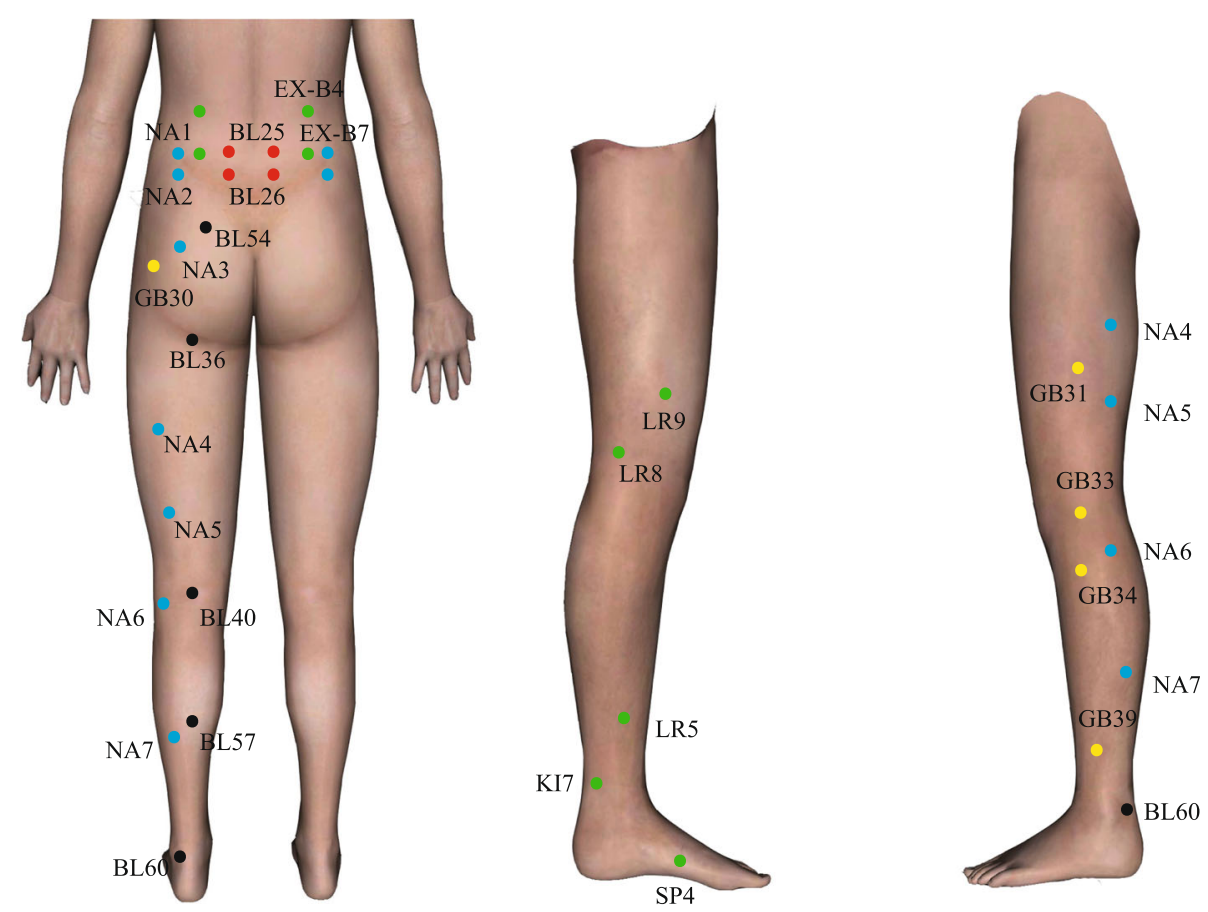

- Obligatory acupoints in DAM group

- Adjunct acupoints on gallbladder meridian in DAM group

- Acupoints in NAM group

- Adjunct acupoints on bladder meridian in DAM group

- Non-acupoints

Fig. 3 Locations of acupoints and non-acupoints. DAM, disease-affected meridians; NAM, non-affected meridians; SA, sham acupuncture

Secondary outcomes include VAS for leg pain and back pain at other time points, Oswestry Disability Index (ODI) [22], the Sciatica Frequency and Bothersomeness Index (SFBI) [23], 36-item Short Form Health Survey (SF-36) [24], straight leg raise test [25], painDETECT questionnaire [26], global perceived recovery on 7-point Likert self-rating scale [27], the Credibility/Expectancy Questionnaire [28], blinding assessment and medicine use. The ODI plays a role in giving a subjective percentage score of function level for patients with low back pain through examining perceived disability in 10 activities of daily living. The SFBI measures the frequency and symptom severity with scores ranging from 0 to 24 respectively.
SF-36 is used to assess the quality of life on eight aspects including physical functioning, bodily pain, role limitations due to physical health problems, personal or emotional problems, emotional well-being, social functioning, energy/fatigue, general health perceptions, and a single item that indicates perceived change in health. PainDETECT questionnaire is used to discern the variation of neuropathic pain as one common method. A 7-point Likert self-rating scale is used to assess the recovery compared to the onset with options from "completely recovered" to "worse than ever." The Credibility/Expectancy Questionnaire is capable to assess the credibility and expectancy of patients. Maintenance of blinding will be determined by

Table 3 Location of non-acupoints in SA group

\begin{tabular}{ll}
\hline Non-acupoints & Location \\
\hline NA 1 (bilateral) & In the lumbar region, 2.5 cun beside Dachangshu (BL25), in the middle of gallbladder meridian and bladder meridian. \\
NA 2 (bilateral) & In the lumbar region, 2.5 cun beside Guanyuanshu (BL26), in the middle of gallbladder meridian and bladder meridian. \\
NA 3 & In the middle of Zhibian (BL54) and Huantiao (GB30) acupoints on the affected leg. \\
NA 4 & 10 cun above the popliteal crease, in the middle of gallbladder meridian and bladder meridian on the affected leg. \\
NA 5 & 5 cun above the popliteal crease, in the middle of gallbladder meridian and bladder meridian on the affected leg. \\
NA 6 & In the middle of Weizhong (BL40) and Yanglingquan (GB34) acupoints on the affected leg. \\
NA 7 & In the middle of Chengshan (BL57) and Waiqiu (GB36) acupoints on the affected leg.
\end{tabular}

One "cun" is defined as the width of the interphalangeal joint of patient's thumb 
Table 4 Schedule of recruitment, interventions, and assessments

\begin{tabular}{|c|c|c|c|c|c|c|c|c|}
\hline & \multirow[t]{2}{*}{ Baseline } & \multicolumn{4}{|c|}{ Treatment period } & & \multicolumn{2}{|c|}{$\begin{array}{l}\text { Follow-up } \\
\text { period }\end{array}$} \\
\hline & & $\begin{array}{l}\text { After the } \\
1 \mathrm{st} \\
\text { treatment }\end{array}$ & $1 \mathrm{w}$ & $2 \mathrm{w}$ & $3 w$ & $4 w$ & $8 w$ & $26 \mathrm{w}$ \\
\hline \multicolumn{9}{|l|}{ Enrolment } \\
\hline Screening & $x$ & & & & & & & \\
\hline Signed informed consent & $x$ & & & & & & & \\
\hline Randomization & $x$ & & & & & & & \\
\hline \multicolumn{9}{|l|}{ Interventions } \\
\hline \multicolumn{9}{|l|}{ DAM group } \\
\hline \multicolumn{9}{|l|}{ NAM group } \\
\hline \multicolumn{9}{|l|}{ SA group } \\
\hline \multicolumn{9}{|l|}{ Assessments } \\
\hline \multicolumn{9}{|l|}{ Primary outcome } \\
\hline VAS for leg pain intensity & $\times$ & & & & & $x$ & & \\
\hline \multicolumn{9}{|l|}{ Secondary outcome } \\
\hline VAS for leg pain intensity & & $\times$ & $\times$ & $\times$ & $x$ & & $\times$ & $x$ \\
\hline ODI & $\times$ & & & $x$ & & $\times$ & $x$ & $x$ \\
\hline VAS for back pain intensity & $x$ & & & $x$ & & $x$ & $x$ & $x$ \\
\hline SFBI & $x$ & & & $x$ & & $x$ & $x$ & $x$ \\
\hline SF-36 & $x$ & & & & & $x$ & $x$ & $x$ \\
\hline Straight leg raise test & $x$ & & & & & $x$ & & $x$ \\
\hline PainDETECT questionnaire & $\times$ & & & & & $\times$ & & $x$ \\
\hline Global perceived recovery & & & & & & $\times$ & & $x$ \\
\hline Medicine use & $x$ & & $x$ & $x$ & $x$ & $\times$ & $x$ & $x$ \\
\hline Credibility assessment & & $x$ & & & & & & \\
\hline Blinding assessment & & & & $x$ & & $x$ & & \\
\hline Adverse events & & $\times$ & $x$ & $\times$ & $\times$ & $\times$ & $\times$ & $x$ \\
\hline
\end{tabular}

DAM disease-affected meridians, NAM non-affected meridians, SA sham acupuncture, $w$ week, VAS visual analogue scale, ODI Oswestry Disability Index, SFBI the Sciatica Frequency and Bothersomeness Index, SF-36 36-item Short Form Health Survey

asking patients to report the group which they believe they had been assigned to. Categories and frequency of medicines use during the trial will be recorded in detail. Detailed arrangements of every outcome are shown in Table 4.

Adverse events will be recorded, valued, and treated timely. Continuous investigation and reexaminations will be conducted until the end of the study. Independent clinical specialists who are blinded to the assignment will judge the severity and relationship between adverse events and acupuncture. Sever adverse events will be reported to the principal investigator and the data and safety monitor board (DSMB) within 24h after occurrence to determine subsequent participation. The occurrence ratio of adverse events will be calculated at the end of the study.

\section{Data management}

Data will be collected clearly and completely on case report forms (CRFs). All original data sources will be preserved including CRFs, informed consents, and inspection results. An independent data manager is in charge of the completed CRFs, he will be asked to input data doubly for proofreading. All data related to the trial will be saved for at least 5 years after publication. Readers will be permitted to access the original data by contacting the corresponding author. Information on patients will remain anonymous including name, age, and telephone number. An independent DSMB is established to ensure the integrity of the research data. The DSMB will review the progress and decide whether a premature closure is needed or not.

\section{Quality control}

The study protocol has been reviewed and revised by experts of acupuncture, orthopedics, statistics, and methodology. All researchers who conduct the study will attend a series of training to promote the implementation. Data will be monitored on 3 levels by the head of sub-centers, researchers from the Beijing University of Chinese Medicine, and the DSMB pre-set for this study. The Research Ethics Committee of Beijing University of Chinese Medicine will audit the study on a regular basis independently. 


\section{Sample size}

As a pilot study, we determine to use a convenience sample instead of a sample size calculation. Ninety patients will be enrolled in this trial, 30 in each group. The data of this pilot trial will be applied to facilitate the calculation of the sample size for a further RCT.

\section{Statistical analysis}

Continuous variables will be described by means and standard deviations or $95 \%$ confidence intervals when following a normal distribution. If not, the data will be shown by medians and interquartile ranges. Categorical variables will be described by frequencies and percentages.

All data will be analyzed following the intention-totreat principle primarily. A per-protocol analysis will be used for primary outcome as sensitivity analysis covering patients who complete at least 10 treatment sessions without obvious violation. Analysis of variance (ANOVA), Kruskal-Wallis $\mathrm{H}$ test, or chi-square test will be used to compare the equilibrium between three groups at baseline. As for outcomes, one-way repeated measures ANOVA or Kruskal-Wallis $H$ test will be applied for continuous variables and chi-square tests or Fisher's exact test will be used for categorical variables as appropriate. Data analyses will be performed using IBM SPSS21.0 with the statistical significance of twotailed $P<0.05$ as exploratory.

\section{Discussion}

Acupuncture might be a potential therapy for sciatica while methodological issues (e.g., lack of random sequence generation, allocation concealment, and blinding) appear frequently in previous studies. Two recent trials provided restricted evidence in favor of acupuncture but both were conducted in a single center with small sample sizes [29, 30]. Generalization of these study results might be limited. This pilot study will be conducted in 6 centers and will develop a full-scale study in order to address the issues.

The mechanism of sciatica may relate to the distortion of the nerve roots and effect of local inflammatory cytokines [1]. Acupuncture is known to exert an analgesic effect through inhibiting cytokine production and activate sympathetic nerve fibers to increase endogenous opioids [31, 32]. It may normalize default mode network activity and modulation of descending pain processing that help treat sciatica [33].

According to the Traditional Chinese Medicine theory, sciatica belongs to disorders of gallbladder meridian and bladder meridian. Acupuncturists tend to choose acupoints on disease-affected meridians in clinical practice which is expected to produce better effect. In our study, acupoints in the DAM group are all attached to the two affected meridians while those in the NAM group are on other meridians less related to sciatica. The comparative results will inspire the optimal acupuncture strategy for sciatica.

The feasibility and efficacy of acupuncture for sciatica will be evaluated in this study. Other strengths involve that it is a multicenter study designed following the Good Clinical Practice guideline [34]. Acupoints are selected based on literature and practical experiences from experts. Our limitations include that we use a subjective primary outcome, while VAS is the commonest method for measuring pain. Based on practical conditions, acupuncturists could not be blinded in this trial but communications will be limited to minimize the impact. Outcomes of this pilot study will be used as evidence for a further RCT subsequently.

\section{Trial status}

Recruitment began on June 9, 2020, and was completed on September 27, 2020. This pilot study (protocol version 2.0, 13 January 2020) is expected to be finished by the end of March 2021.

\section{Amendments \\ Change in study centers}

Affected by the COVID-19, the Guang'anmen Hospital failed to get administrative support from government and was not permitted to recruit patients. To ensure recruitment and reach the target sample size, we had to add 3 other centers: the Union Hospital, Tongji Medical College, Huazhong University of Science and Technology; the Central Hospital of Wuhan, Tongji Medical College, Huazhong University of Science and Technology; and the Shenzhen Hospital, Beijing University of Chinese Medicine. Finally, 6 hospitals are conducting this pilot study. The final center was confirmed on 26 June, 2020.

\section{Change in inclusion criteria}

We planned to enroll patients with acute, subcute, and chronic sciatica initially. However, as many patients chose to stay at home rather than visit doctors under the impact of the COVID-19 pandemic, we realized it could be very hard to enroll patients with acute sciatica. After discussions with experts, we finally decided to enroll patients with sciatica for 1 month or longer. This change was made on May 28, 2020.

\section{Change in exclusion criteria}

The length of time for patients receiving acupuncture treatment previously was adjusted from 1 year to 6 months in exclusion criteria. This change was made based on advice from methodological experts who suggested that it would be enough to exclude the lasting 
effects of acupuncture in 6 months. This change was made on May 28, 2020.

\section{Change in secondary outcome}

Based on suggestions from experts, we have added "the Sciatica Frequency and Bothersomeness Index" as an additional outcome to measure the frequency and bothersomeness for sciatica. It is commonly used in previous studies with good reliability [24]. This change was made on May 28, 2020.

\section{Supplementary Information}

The online version contains supplementary material available at https://doi. org/10.1186/s13063-020-04961-4

Additional file 1. SPIRIT 2013 Checklist: Recommended items to address in a clinical trial protocol and related documents.

\section{Abbreviations}

DAM: Disease-affected meridians; NAM: Non-affected meridians; SA: Sham acupuncture; RCT: Randomized controlled trial; CONSORT: Consolidated Standards of Reporting Trials; STRICTA: The Standards for Reporting Interventions in Clinical Trials of Acupuncture guidelines; SPIRIT: The Standard Protocol Items; DSMB: Data and safety monitor board; CRF: Case report form; MRI: Magnetic resonance imaging; $\mathrm{CT}$ : Computed tomography VAS: Visual analogue scale; ODI: Oswestry Disability Index; SFBI: The Sciatica Frequency and Bothersomeness Index; SF-36: 36-item Short Form Health Survey; ANOVA: Analysis of variance

\section{Acknowledgements}

The authors would like to thank Dr. Zhi-Shun Liu who contributed to the design of acupuncture procedure.

\section{Authors' contributions}

GXS, CZL, JLL, and FTY designed and developed the trial. GXN, GWC, WJW, $X Q Z$, and XLM offered administrative support. HYF, XCZ, JL, YFW, BZ, HLZ, and $\mathrm{XHZ}$ are in charge of recruitment and treatment. JFT, LQW, and JWY provided methodological recommendations. FTY, GXS, and CZLu drafted and critically revised the manuscript. All authors have read and approved the final manuscript.

\section{Funding}

The National Key Research and Development Program of China supports this work (No.2019YFC1712101). The sponsor has no role in study design, data analysis, or the decision to submit the report for publication.

\section{Availability of data and materials}

Data sharing is not applicable to this article as no datasets were generated or analyzed during the current study.

\section{Ethics approval and consent to participate}

The Research Ethics Committee of Beijing University of Chinese Medicine has approved the study with the approval number of 2020BZHYLL0105. The methods are carried out per the Declaration of Helsinki. Written informed consent will be obtained from all patients included prior to randomization in the study. Any modifications to the protocol will be reported. During study period, consultations related or unrelated to sciatica will be offered freely to patients. When the study is finished, an additional personal advice will be given based on their conditions. No other compensation will be offered.

\section{Consent for publication}

Not applicable.

\section{Competing interests}

The authors declare that they have no competing interests.

\section{Author details}

International Acupuncture and Moxibustion Innovation Institute, Beijing University of Chinese Medicine, Beijing 100029, China. ${ }^{2}$ Acupuncture Research Center, School of Acupuncture-Moxibustion and Tuina, Beijing University of Chinese Medicine, Beijing 100029, China. ${ }^{3}$ Nanjing University of Chinese Medicine, Nanjing 210023, China. ${ }^{4}$ Department of Acupuncture, Union Hospital, Tongji Medical College, Huazhong University of Science and Technology, Wuhan 430022, China. ${ }^{5}$ Department of Rehabilitation, The Central Hospital of Wuhan, Tongji Medical College, Huazhong University of Science and Technology, Wuhan 430014, China. ${ }^{6}$ Shenzhen Hospital, Beijing University of Chinese Medicine, Shenzhen 518100, China. ${ }^{7}$ Pain Medicine Center, Peking University Third Hospital, Beijing 100191, China. ${ }^{8}$ Affiliated Hospital of Nanjing University of Chinese Medicine, Nanjing 210029, China. ${ }^{9}$ Department of Traditional Chinese Medicine, Peking University of Third Hospital, Beijing 100191, China. ${ }^{10}$ Department of acupuncture, Dongzhimen Hospital Affiliated to Beijing University of Chinese Medicine, Beijing 100700, China.

Received: 24 May 2020 Accepted: 9 December 2020

Published online: 07 January 2021

\section{References}

1. Ropper AH, Zafonte RD. Sciatica. N Engl J Med. 2015;372(13):1240-8

2. Konstantinou K, Dunn KM. Sciatica: review of epidemiological studies and prevalence estimates. Spine (Phila Pa 1976). 2008;33(22):2464-72.

3. Deyo RA, Mirza SK. Clinical practice. Herniated lumbar intervertebral disk. N Engl J Med. 2016;374(18):1763-72.

4. Jensen RK, Kongsted A, Kjaer P, et al. Diagnosis and treatment of sciatica. BMJ. 2019;367:16273.

5. Valat JP, Genevay S, Marty M, et al. Sciatica. Best Pract Res Clin Rheumatol. 2010;24:241-52.

6. van Tulder M, Peul W, Koes B. Sciatica: what the rheumatologist needs to know. Nat Rev Rheumatol. 2010;6(3):139-45.

7. Pinto RZ, Verwoerd A, Koes BW. Which pain medications are effective for sciatica (radicular leg pain). BMJ. 2017:359:j4248.

8. Kreiner DS, Hwang SW, Easa JE, et al. An evidence-based clinical guideline for the diagnosis and treatment of lumbar disc herniation with radiculopathy. Spine J. 2014;14(1):180-91.

9. Page J, Moisi M, Oskouian RJ. Lumbar epidural hematoma following interlaminar fluoroscopically guided epidural steroid injection. Reg Anesth Pain Med. 2016:41(3):402-4.

10. Weber $\mathrm{H}$, Holme I, Amlie E. The natural course of acute sciatica with nerve root symptoms in a double-blind placebo-controlled trial evaluating the effect of piroxicam. Spine (Phila Pa 1976). 1993;18(11):1433-8.

11. Vickers AJ, Vertosick EA, Lewith $G$, et al. Acupuncture for chronic pain: update of an individual patient data meta-analysis. J Pain. 2018;19(5):455-74.

12. Qin Z, Liu X, Wu J, et al. Effectiveness of acupuncture for treating sciatica: a systematic review and meta-analysis. Evid Based Complement Alternat Med. 2015;2015:425108

13. Ji $M$, Wang $X$, Chen $M$, et al. The efficacy of acupuncture for the treatment of sciatica: a systematic review and meta-analysis. Evid Based Complement Alternat Med. 2015:2015:192808.

14. Zhao L, Li DH, Zheng H, et al. Acupuncture as adjunctive therapy for chronic stable angina: a randomized clinical trial. JAMA Intern Med. 2019; 179(10):1388-97.

15. Yang J, Zeng F, Feng $Y$, et al. A PET-CT study on the specificity of acupoints through acupuncture treatment in migraine patients. BMC Complement Altern Med. 2012;12:123.

16. Schulz KF, Altman DG, Moher D, et al. CONSORT 2010 statement: updated guidelines for reporting parallel group randomized trials. Obstet Gynecol. 2010;115(5):1063-70.

17. MacPherson $H$, Altman DG, Hammerschlag $R$, et al. Revised STandards for Reporting Interventions in Clinical Trials of Acupuncture (STRICTA): extending the CONSORT statement. PLOS Med. 2010;7(6):e1000261.

18. Collins SL, Moore RA, McQuay HJ. The visual analogue pain intensity scale: what is moderate pain in millimetres? Pain. 1997;72:95-7.

19. Zhang $L$, Zhou J, Wang $P$, et al. Study on the rule of acupoint selection in the treatment of sciatica with acupuncture. J Clin Acupunct Moxibustion. 2020:(01):53-6. https://kns.cnki.net/kcms/detail/detail.aspx?FileName=ZJLC2 02001015\&DbName=CJFQ2020. 
20. Qiu T, Li L. Discussion on the Chinese edition of the WHO standard acupuncture point locations in the Western Pacific region. Zhongguo Zhen Jiu. 2011;31(9):827-30.

21. Yang XG, Ying $L$, Tian XP, et al. Comments on selection of non-acupoints beyond meridians in studies of acupuncture and moxibustion. J Tradit Chin Med. 2010;30(4):309-13.

22. Fairbank JC, Couper J, Davies JB, et al. The Oswestry low back pain disability questionnaire. Physiotherapy. 1980;66(8):271-3.

23. Brazier JE, Harper R, Jones NM, et al. Validating the SF-36 health survey questionnaire: new outcome measure for primary care. BMJ. 1992;305: 160-4.

24. Patrick DL, Deyo RA, Atlas SJ, et al. Assessing health-related quality of life in patients with sciatica. Spine. 1995;20:1899-908.

25. Hsieh CY, Walker JM, Gillis K. Straight-leg-raising test. Comparison of three instruments. Phys Ther. 1983;63(9):1429-33.

26. Freynhagen R, Baron R, Gockel U, et al. painDETECT: a new screening questionnaire to identify neuropathic components in patients with back pain. Curr Med Res Opin. 2006;22:1911-20.

27. Peul WC, van Houwelingen $H C$, van den Hout WB, et al. Surgery versus prolonged conservative treatment for sciatica. N Engl J Med. 2007;356(22): 2245-56.

28. Devilly GJ, Borkovec TD. Psychometric properties of the credibility/ expectancy questionnaire. J Behav Ther Exp Psychiatry. 2000;31:73-86.

29. Huang Z, Liu S, Zhou J, et al. Efficacy and safety of acupuncture for chronic discogenic sciatica, a randomized controlled sham acupuncture trial. Pain Med. 2019;20(11):2303-10.

30. Zhang $X$, Wang $Y$, Wang $Z$, et al. A randomized clinical trial comparing the effectiveness of electroacupuncture versus medium-frequency electrotherapy for discogenic sciatica. Evid Based Complement Alternat Med. 2017;2017:9502718.

31. Torres-Rosas R, Yehia G, Peña G, et al. Dopamine mediates vagal modulation of the immune system by electroacupuncture. Nat Med. 2014; 20(3):291-5.

32. Zhang R, Lao L, Ren $K$, et al. Mechanisms of acupunctureelectroacupuncture on persistent pain. Anesthesiology. 2014;120(2):482-503.

33. Liu CH, Yeh TC, Kung YY, et al. Changes in resting-state functional connectivity in nonacute sciatica with acupuncture modulation: a preliminary study. Brain Behav. 2020;10(2):e01494.

34. Grimes DA, Hubacher D, Nanda K, et al. The good clinical practice guideline: a bronze standard for clinical research. Lancet. 2005;366(9480):172-4.

\section{Publisher's Note}

Springer Nature remains neutral with regard to jurisdictional claims in published maps and institutional affiliations.

Ready to submit your research? Choose BMC and benefit from:

- fast, convenient online submission

- thorough peer review by experienced researchers in your field

- rapid publication on acceptance

- support for research data, including large and complex data types

- gold Open Access which fosters wider collaboration and increased citations

- maximum visibility for your research: over $100 \mathrm{M}$ website views per year

At $\mathrm{BMC}$, research is always in progress.

Learn more biomedcentral.com/submissions 